Psychotherapeut 2017 $62: 161-163$

DOI 10.1007/s00278-017-0187-7

Online publiziert:4. Mai 2017

๑) Springer Medizin Verlag Berlin 2017

CrossMark

\author{
Anja Hilbert ${ }^{1} \cdot$ Silja Vocks ${ }^{2}$ \\ ${ }^{1}$ Integriertes Forschungs- und Behandlungszentrum AdipositasErkrankungen, Medizinische Psychologie \\ und Medizinische Soziologie, Psychosomatische Medizin und Psychotherapie, Universitätsmedizin \\ Leipzig, Leipzig, Deutschland \\ ${ }^{2}$ Institut für Psychologie, Fachgebiet Klinische Psychologie und Psychotherapie, Universität Osnabrück, \\ Osnabrück, Deutschland
}

\title{
Aktuelle Behandlungsansätze für Essstörungen
}

ten [AWMF] 2010) empirisch gestützte Behandlungsempfehlungen abgeleitet, die außerdem im Rahmen einer $\mathrm{Pa}$ tientenleitlinie Betroffenen und deren Angehörigen zugänglich gemacht wurden (AWMF 2015). Evidenzbasierte Leitlinien müssen jeweils nach fünf Jahren wieder an den aktuellen Stand der Forschung angepasst werden.

In dem vorliegenden Schwerpunktheft Psychotherapie bei Essstörungen berichten daher Herpertz und HerpertzDahlmann über den Status quo zur Revision der evidenzbasierten S3-Leitlinie Diagnostik und Therapie der Essstörungen, deren Erscheinen für 2017 geplant ist. Weiterhin in diesem Schwerpunktheft zusammengestellt ist aktuelle, für die Diagnostik und Behandlung verschiedener Essstörungen relevante Forschung.

Ein wesentliches Moment in der Entstehung und Aufrechterhaltung von Essstörungen sowie verschiedener anderer psychischen Störungen (z. B. körperdysmorphe Störung) ist ein negatives Körperbild. Ein negatives Körperbild kann sich in verzerrten Wahrnehmungsprozessen, bezogen auf die eigene Figur, Unzufriedenheit mit dem eigenen Körper sowie einer erhöhten Relevanz von Figur oder Gewicht für das Selbstwertgefühl, oder körperbezogenem Vermeidungs- und Kontrollverhalten manifestieren. Während für die Anorexia und Bulimia nervosa kognitiv-affektive Aspekte einer Körperbildstörung in den Diagnosekriterien (APA 2015) enthalten sind, ist die Körperbildstörung für die Diagnosestellung bei der Binge-Eating-Störung nicht relevant; sie wurde jedoch als Indikator für den Schweregrad dieser Störung vorgeschlagen (Grilo et al. 2015). Körperbildstörungen sind zudem ein wesentlicher Ansatzpunkt für die Psychotherapie bei Essstörungen, wie in verschiedenen Manualen dargestellt (z. B. Hilbert und Tuschen-Caffier 2010; Legenbauer und Vocks 2014; Tuschen-Caffier und Florin 2012; Vocks und Legenbauer 2010). Eine reliable und valide Eingangs-, Verlaufs- und evaluative Diagnostik der Körperbildstörung ist daher Voraussetzung für eine fundierte Behandlung. In dem vorliegenden Schwerpunktheft geben Steinfeld et al. einen Überblick über die deutschsprachig zur Verfügung stehenden Instrumente zur Erfassung der diversen oben genannten Facetten des Körperbildes.

Aufgrund zunehmenden Kostendrucks im Gesundheitswesen wurden über die vergangenen Jahrzehnte hinweg strukturierte Selbsthilfeprogramme für Essstörungen entwickelt und erforscht. Damit sind Programme gemeint, die auf etablierten therapeutischen Ansätzen wie der kognitiven Verhaltenstherapie fußen, als Buch (d. h. für Bibliotherapie) oder elektronisch (z. B. als Internetprogramm) manualisiert wurden und - in den meisten Fällen - durch einen Therapeuten oder Trainer individuell angeleitet werden (Wilson und Zandberg 2012). Diese strukturierten Programme sind also von gängigen reinen unstrukturierten Selbsthilfegruppen zu unterscheiden. Es wird angenommen, dass strukturierte Selbsthilfeprograme die Inanspruchnahme von Behandlungen für Essstörungen verbessern können, für bestimmte $\mathrm{Pa}$ tienten sogar als alleinige Behandlung ausreichen und somit der aktuellen lichen Medizinischen Fachgesellschaf- 
Unterversorgung von Essstörungen abhelfen können (Kessler et al. 2013). Erste Belege für die Kosteneffektivität liegen vor (Lynch et al. 2010). Strukturierte Selbsthilfeprogramme haben bereits in die Behandlungsempfehlungen der S3Leitlinie Diagnostik und Therapie der Essstörungen (AWMF 2010) Eingang gefunden, und es ist zu erwarten, dass dies auch in der anstehenden Revision der Leitlinie der Fall sein wird. Beintner und Jacobi geben in einer Metaanalyse einen aktuellen Überblick über die Wirksamkeit von strukturierten Selbsthilfeprogrammen in der Behandlung von Essstörungen. Forschung zu diesem Thema ist zentral, um solche Selbsthilfeprogramme - bei vorhandenem Wirksamkeitsnachweis - als Angebot der Regelversorgung im Gesundheitswesen verankern zu können.

Essstörungen werden zunehmend als Störungen der Selbstregulation oder dem heutzutage dominanten neurowissenschaftlichen Forschungsparadigma folgend - als neurokognitive oder -behaviorale Störungen verstanden. Verschiedene Studien belegen dementsprechend Veränderungen in Aufmerksamkeit und mentaler Repräsentation (z. B. von nahrungs- oder körperbezogenen Stimuli), Ansprechen auf Belohnung, Kontrolle von Handlungsimpulsen sowie Emotionswahrnehmung und -regulation (Kittel et al. 2015; Wu et al. 2016). Inwiefern diese Prozesse den Essstörungen ursächlich zugrunde liegen und für sie spezifisch sind, und inwiefern Interventionen, die auf eine Reduktion dieser neurokognitiven Auffälligkeiten abzielen, geeignet sind, um Essstörungen zu behandeln, ist allerdings noch unklar. Svaldi et al. fassen am Beispiel der Binge-Eating-Störung den aktuellen Forschungsstand zu dieser Thematik zusammen und stellen mögliche Zusammenhänge zwischen den einzelnen Prozessen und der Essanfallssymptomatik in einem anschaulichen Störungsmodell dar.

In diesem Kontext sind eine Entwicklung und Testung von Behandlungsansätzen, die auf eine Förderung der Selbstregulation abzielen, von hoher Relevanz. Verschiedene einzelne Interventionen, z. B. die Approach Bias Modification zur Behandlung einer erhöhten
Belohnungssensitivität für Nahrungsreize (z. B. Brockmeyer et al. 2015), und komplexere Programme, wie die Kognitive Remediationstherapie (z. B. Lindvall Dahlgren und Rø 2014), befinden sich derzeit in Entwicklung und Evaluation für Essstörungen. Ein weiterer Ansatz, das direkt an biologischen Signalen ansetzende Biofeedback, hat sich für verschiedene psychische Störungen bereits als wirksam erwiesen, darunter die Aufmerksamkeitsdefizit-/ Hyperaktivitätsstörung (Cortese et al. 2016). Biofeedback beinhaltet Methoden, mittels derer der Patient noninvasiv eine Echtzeitrückmeldung über Körpersignale erhält, die i. Allg. nicht wahrnehmbar sind (z. B. Gehirnaktivität, elektrodermale Hautleitfähigkeit). Blume et al. geben in diesem Schwerpunktheft einen Überblick über den aktuellen Forschungsstand zu Methoden und Wirksamkeit des Biofeedbacks bei Ess- und Gewichtsstörungen.

Obwohl zur Bedeutung von familien-, partnerschafts- und berufsbezogenen Faktoren im Kontext der Entstehung und Aufrechterhaltung von Essstörungen mittlerweile zahlreiche - wenn auch widersprüchliche - Forschungsergebnisse vorliegen, wurde der Einfluss dieser Variablen auf den Therapieerfolg bisher kaum untersucht. Daher wird in dem Artikel von Teufel et al. die in der „Anorexia Nervosa Treatment Of Outpatients“-Studie (Zipfel et al. 2014) untersuchte Stichprobe in Bezug auf soziodemografische Faktoren mit der Allgemeinbevölkerung verglichen, und der Einfluss dieser Variablen auf den Therapieerfolg wird untersucht.

Bei zunehmenden bariatrischen Operationen sind Psychotherapeuten immer mehr mit Patienten konfrontiert, die ihre extreme Adipositas (d.h. Fettleibigkeit mit Body-Mass-Index $\geq 40 \mathrm{~kg} / \mathrm{m}^{2}$ oder $\geq 35 \mathrm{~kg} / \mathrm{m}^{2}$ mit Folgeerkrankungen der Adipositas) durch operative Eingriffe wie den Roux-en-Y-Magenbypass oder den Schlauchmagen behandeln lassen möchten oder sich einem solchen Eingriff unterzogen haben. Entsprechend der S3-Leitlinie Prävention und Therapie der Adipositas (AWMF 2014) ist Voraussetzung für die Kostenübernahme einer bariatrischen Therapie ein psychologi- sches Gutachten, mit dem psychosoziale Kontraindikationen wie eine psychische Instabilität ausgeschlossen werden sollen (de Zwaan et al. 2007). Zusätzlich, und dies ist in der gültigen Leitlinie noch nicht festgelegt, benötigen Patienten mit Psychopathologie vor und nach der Operation eine geeignete interdisziplinäre Vor- und Nachsorge und/oder Psychotherapie. Psychische Störungen wie Essstörungen, v. a. die Binge-EatingStörung, depressive und Angststörungen treten bei extremer Adipositas gehäuft auf und verringern den Gewichtsreduktionserfolg durch bariatrische Therapie (Müller et al. 2013). Wild et al. beschreiben in diesem Schwerpunktheft einen durch Videokonferenzen unterstützten Ansatz zur Nachsorge nach bariatrischer Operation und dessen Wirkungen auf das Essverhalten.

Die Zusammenstellung von Beiträgen zu aktuellen Entwicklungen in der Psychotherapie bei Essstörungen in diesem Schwerpunktheft zeigt auf, wie aktuelle Forschung das Störungsverständnis von Essstörungen und Ansätze zur Diagnostik und Therapie beeinflusst und neue Herausforderungen für psychotherapeutisches Handeln mit sich bringt. Sie birgt zugleich die Chance, die Wirksamkeit von Psychotherapie bei Essstörungen weiter zu erhöhen und die Versorgung der Betroffenen zu verbessern.

\section{Korrespondenzadresse}

\section{Prof. Dr. A. Hilbert}

Integriertes Forschungs- und Behandlungszentrum AdipositasErkrankungen, Medizinische Psychologie und Medizinische Soziologie, Psychosomatische Medizin und Psychotherapie, Universitätsmedizin Leipzig Philipp-Rosenthal-Str. 27, 04103 Leipzig, Deutschland

anja.hilbert@medizin.uni-leipzig.de

Danksagung. Die Autorinnen danken Lisa Opitz, B.Sc. für ihre editorische Unterstützung des Manuskripts.

Förderung. A. Hilbert wird gefördert durch das Bundesministerium für Bildung und Forschung, FKZ $01 \mathrm{E} 01001$.

Interessenkonflikt. A. Hilbert und S. Vocks geben an, dass kein Interessenkonflikt besteht. 


\section{Literatur}

American Psychiatric Association (APA) (2015) Diagnostisches und statistisches Manual psychischer Störungen DSM-5. Hogrefe, Göttingen

Arbeitsgemeinschaft der Wissenschaftlichen Medizinischen Fachgesellschaften (AWMF) (2010) S3Leitlinie „Diagnostikund Therapie der Essstörungen". http://www.awmf.org/leitlinien/detail/ll/ 051-026.html.Zugegriffen: 15. Feb 2017

AWMF (2014) S3-Leitlinie „Prävention und Therapie der Adipositas". http://www.awmf.org/leitlinien/ detail/l//050-001.html. Zugegriffen: 15. Feb 2017

AWMF (2015) Patientenleitlinie „Diagnostik und Behandlung von Essstörungen“.http://www.awmf org/leitlinien/detail/ll/051-026.html. Zugegriffen: 15. Feb 2017

Brockmeyer T, Hahn C, Reetz C, Schmidt U, Friederich HC (2015) Approach bias modification in food craving - a proof-of-concept study. Eur Eat Disord Rev 23:352-360

Cortese S, Ferrin M, Brandeis D, Holtmann M, Aggensteiner P, Daley D, Santosh P, Simonoff $E$, Stevenson J, Stringaris A, Sonuga-Barke EJ, European ADHD Guidelines Group (EAGG) (2016) Neurofeedback for attention-deficit/ hyperactivity disorder: meta-analysis of clinical and neuropsychological outcomes from randomized controlled trials. J Am Acad Child Adolesc Psychiatry 55:444-455

de Zwaan M, Wolf AM, Herpertz S (2007) Psychosomatische Aspekte der Adipositaschirurgie: Was ist empirisch gesichert? Dtsch Arztebl 104:A2577-A2583

Fairburn CG, Walsh BT (2002) Atypical eating disorders (eating disorder not otherwise specified). In: Fairburn CG, Brownell KD (Hrsg) Eating disorders and obesity: a comprehensive handbook. Guilford Press, New York, S171-177

Grilo CM, Ivezaj V, White MA (2015) Evaluation of the DSM-5 severity indicator for binge eating disorder in a clinical sample. Behav Res Ther 71:110-114

Hilbert A, Tuschen-Caffier B (2010) Essanfälle und Adipositas: ein Manual zur kognitiv-behavioralen Therapie der Binge-Eating-Störung. Hogrefe, Göttingen

Kessler RC, Berglund PA, Chiu WT, Deitz AC, Hudson Jl, Shahly V, Aguilar-Gaxiola S, Alonso J, Angermeyer MC, Benjet $C$, Bruffaerts $R$, de Girolamo G, de Graaf R, Maria HJ, KovessMasfety V, O'Neill S, Posada-Villa J, Sasu C, Scott K, Viana MC, Xavier M (2013) The prevalence and correlates of binge eating disorder in the World Health Organization world mental health surveys. Biol Psychiatry 73:904-914

Kittel R, Brauhardt A, Hilbert A (2015) Cognitive and emotional functioning in binge-eating disorder: a systematic review. Int J Eat Disord 48:535-554

Legenbauer T, Vocks S (2014) Manual der kognitiven Verhaltenstherapie bei Anorexie und Bulimie. Springer, Berlin

Lindvall Dahlgren C, Rø $\emptyset$ (2014) A systematic review of cognitive remediation therapy for anorexia nervosa - development, current state and implications for future research and clinical practice. J Eat Disord 2:26

Lynch FL, Striegel-Moore RH, Dickerson JF, Perrin N, Debar L, Wilson GT, Kraemer HC (2010) Costeffectiveness of guided self-help treatment for recurrent binge eating. J Consult Clin Psychol 78:322-333
Müller A, Mitchell JE, Sondag C, de Zwaan M (2013) Psychiatric aspects of bariatric surgery. Curr Psychiatry Rep 15:397

Tuschen-Caffier B, Florin I (2012) Teufelskreis Bulimie: ein Manual zur psychologischen Therapie. Hogrefe, Göttingen

Vocks S, Legenbauer T (2010) Körperbildtherapie bei Anorexia und Bulimia nervosa. Ein kognitivverhaltenstherapeutisches Behandlungsprogramm. Hogrefe, Göttingen

Weltgesundheitsorganisation (2016) Internationale statistische Klassifikation der Krankheiten und verwandter Gesundheitsprobleme. 10. Revision - German Modification (ICD-10-GM, Version 2017). Deutscher Ärzteverlag, Köln

Wilson GT, Zandberg LJ (2012) Cognitive-behavioral guided self-help for eating disorders: effectiveness and scalability. Clin Psychol Rev 32:343-357

Wu M, Brockmeyer T, Hartmann $M$, Skunde $M$, Herzog W, Friederich HC (2016) Reward-related decision making in eating and weight disorders: a systematic review and meta-analysis of the evidence from neuropsychological studies. Neurosci Biobehav Rev 61:177-196

Zipfel S, Wild B, Groß G, Friederich HC, Teufel M, Schellberg D, Giel KE, de Zwaan M, Dinkel A, Herpertz S, Burgmer M, Löwe B, Tagay S, von Wietersheim J, Zeeck A, Schade-Brittinger C, Schauenburg $\mathrm{H}$, Herzog W, ANTOP study group (2014) Focal psychodynamic therapy, cognitive behaviour therapy, and optimised treatment as usual in outpatients with anorexia nervosa (ANTOP study): randomised controlled trial. Lancet 383:127-137

\section{Schwerpunktthemen}

Das Herausgebergremium der Zeitschrift Psychotherapeut lädt Autorinnen und Autoren ein, an den geplanten Schwerpunkten mitzuarbeiten und geeignete Manuskripte einzureichen. Diese werden dem üblichen Reviewverfahren unterzogen. Darüber hinaus freuen wir uns über die Zusendung freier (unaufgeforderter) Originalia zu selbstgewählten Themen.

Bitte schicken Sie Ihren Beitrag an die Redaktion:

Regine.Karcher-Reiners@springer.com

Die Schwerpunktplanung ist vorläufig und kann kurzfristig umdisponiert werden.

Heft 6/2017: Psychotherapeutische Bezüge der Psychokardiologie

(Deadline 30.06.2017)

Heft 1/2018: Herausforderungen und Möglichkeiten der Digitalisierung

(Deadline 30.08.2017)

Heft 2/2018: Angst, Ressentiment, Hoffnung. Hass, Fanatismus, Versöhnung.

Lindauer Psychotherapiewochen 2017 (Deadline 30.10.2017)

Heft 3/2018: Angst (Deadline 30.12.2017)

Heft 4/2018: Geschichtsvergessenheit (Deadline 30.02.2018)

Heft 5/2018: Sexualität und sexuelle Störungen (Deadline 30.04.2018)

Heft 6/2018: Studium zur Approbation (Deadline 30.06.2018) 